\title{
SYMPTOMATIC PRIMARY HIV INFECTION IN A 49-YEAR-OLD MAN WHO HAS SEX WITH MEN: BEWARE OF THE WINDOW PHASE
}

\author{
H E van Oosten ${ }^{1}$, M Damen ${ }^{2,3}$, H JC de Vries (h.j.devries@amc.nl)1,4,5 \\ 1. STI Outpatient Clinic, Cluster Infectious Diseases, Municipal Health Service of Amsterdam, Amsterdam, the Netherlands \\ 2. Public Health Laboratory, Cluster Infectious Diseases, Municipal Health Service Amsterdam, Amsterdam, the Netherlands \\ 3. Department of Medical Microbiology, Onze Lieve Vrouwe Gasthuis general hospital, Amsterdam, the Netherlands \\ 4. Department of Dermatology, Academic Medical Center, University of Amsterdam, Amsterdam, the Netherlands \\ 5. Centre for Infectious Diseases Control, National Institute for Public Health and the Environment (Rijksinstituut voor \\ Volksgezondheid en Milieu, RIVM), Bilthoven, the Netherlands
}

This article was published on 9 December 2009.

Citation style for this article: van Oosten HE, Damen M, de Vries HJ. Symptomatic primary HIV infection in a 49 -year-old man who has sex with men: beware of the window phase. Euro Surveill. 2009;14(48):pii=19424. Available online: http://www.eurosurveillance.org/ViewArticle.aspx?ArticleId=19424

A 49-year-old man with a history of receptive unprotected anal intercourse with multiple anonymous men presented with a symptomatic primary HIV infection. Upon his initial visit the rapid HIV antibody screening test was negative but a p24 antigen test suggested a highly infectious phase in the HIV infection. An immunoblot assay confirmed the HIV diagnosis only 14 days later. Recent infections are characterised by a highly infectious phase and, if gone unnoticed, can have a large contribution to the ongoing transmission of HIV. Healthcare providers should be aware of primary HIV infection and the pitfalls in its diagnosis.

\section{Case report}

A 49-year-old man visited the Amsterdam STI outpatient clinic in the fall of 2009 with fever, malaise, generalised rash, anal itching and rectal discharge after unprotected receptive anal and oral intercourse with an anonymous partner in a gay cinema one week before. In the last six months he had engaged in protected and unprotected receptive anal and oral intercourse with 15 different male partners. The last HIV test was performed one year ago and gave a negative test result. He was treated for a syphilis infection with unknown duration in 2004.

The patient presented with fever (39.2 degrees Celsius), generalised erythemato-squamous rash, generalised lymphadenopathy, erythematous pharyngeal mucosa and perianal ulceration. Anoscopy revealed erythematous rectal mucosa which bled easily upon manipulation.

\section{Laboratory findings and follow up}

The patient was routinely screened for syphilis, Neisseria gonorrhoeae, Chlamydia trachomatis, HIV and hepatitis B, according to the standard protocol described before [1]. Awaiting definite results of syphilis serology (Treponema pallidum particle agglutination assay, TPPA), secondary stage syphilis was excluded with a directly performed Rapid Plasma Reagin card test (RPR nosticon II, bioMerieux, France). The direct RPR card test was non reactive in both $1: 1$ and 1:32 serum dilutions. Also, a point of care rapid HIV ELISA antibody test (Determine HIV 1/2, Inverness Medical, United Kingdom) was negative. A Gram-stained smear obtained during anoscopy from the rectal mucosa showed more than 10 polymorphic nucleated leucocytes (PMNL) per high power field without intracellular diplococci, suggestive for a non-specific proctitis. Based on these preliminary results the patient was treated with doxycycline $100 \mathrm{mg}$ twice a day and requested to return seven days later for additional results and follow-up.

From the rectal site a $N$. gonorrhoeae strain was cultivated which showed resistance to ciprofloxacin and tetracycline, intermediate resistance to penicillin and reduced susceptibility to cefotaxime (with a minimal inhibitory concentration [MIC] of $0.19 \mu \mathrm{g} / \mathrm{ml}$ ). C. trachomatis infection was excluded with a nucleic acid amplification assay (Aptima combo, Tigris, Genprobe, United States) performed on a rectal swab and a first void urine sample. Syphilis serology was in concordance with a past, adequately treated, syphilis infection (TPPA 1:160, RPR negative, FTAabsorption positive, Treponema pallidum immunoblot positive). A polymerase chain reaction of the swab taken from the rectal ulcer was negative for Treponema pallidum, herpes simplex virus type 1 and 2 and varicella zoster virus [2,3]. Hepatitis B serology was indicative for a past recovered infection (hepatitis B core antibody positive, hepatitis B surface antigen negative). The rectal gonorrhoea infection was additionally treated with 500 mg ceftriaxone in one intra-muscular dose, while the doxycycline regimen was discontinued.

An HIV antigen/antibody (Ag/Ab) COMBO assay (AxSYM HIV Ag/ Ab Combo, Abbott, United States) performed on the serum sample obtained during the initial visit showed a weak positive result (1.22 $\mathrm{s} / \mathrm{co}$ ). Since we suspected a primary symptomatic HIV infection, and according to routine laboratory procedures, the serum was further tested for the presence of HIV p24 antigen (VIDAS HIV P24, bioMérieux, France), and showed a positive result $(31.2 \mathrm{pg} / \mathrm{ml})$. The immunoblot analysis (INNO-LIA HIV I/II Score, Innogenetics, Belgium) was negative for all antigen bands. Based on these test results from the initial visit, the official criteria for an HIV diagnosis could not be met. Upon return seven days after the initial visit, the skin symptoms, proctitis complaints and fever had subsided. Again, the HIV Ag/Ab COMBO assay was weak positive (1.43 s/ 
co), the HIV p24 antigen load was $17.6 \mathrm{pg} / \mathrm{ml}$ and all bands in the immunoblot were negative. Only 14 days after the initial visit the HIV Ag/Ab COMBO assay turned positive ( $5.2 \mathrm{~s} / \mathrm{co}$ ), the HIV p24 antigen load was $64.6 \mathrm{pg} / \mathrm{ml}$. Immunoblot analysis showed positive bands for anti-GP41 and anti-p24 which confirmed the HIV infection. The patient was referred to an HIV treatment centre for further monitoring and care.

Repeated serologic testing for syphilis 14 days after the initial visit showed the same results as before (TPPA 1:160, RPR negative) consistent with an adequately treated syphilis infection. The negative repeat TPPA may still miss an early syphilis infection but, given the negative polymerase chain reaction for Treponema pallidum of the swab taken from the rectal ulcer at the initial visit, an early syphilis infection was unlikely.

\section{Discussion and conclusion}

We report a 49-year-old male patient with marked exanthema, genital ulcer, and fever suspected for a primary HIV infection. A rapid HIV antibody test was negative at the initial visit. We could only establish the diagnosis 14 days after the initial visit, when the immunoblot turned positive for antibodies against HIV-1.

Primary HIV infections play a key role in the spread of HIV $[4,5]$. During this first phase, the viral load in blood plasma is high $[6,7]$ and patients can shed high concentrations of HIV through semen, saliva and blood $[8,9]$. The Rakai study in Uganda showed that the average per-act transmission rate during primary infection equalled 0.03604 per sex encounter compared to 0.00084 per sex encounter for chronic HIV infections. In that same study, primaryphase transmission accounted for $89.1 \%$ of all transmission events in the first 20 months of follow-up [10,11]. It is possible that primary-phase transmission is even more frequent for MSM since anal intercourse contributes to transmission risk.

Patients with a primary HIV infection are often unaware of their (positive) HIV status and assuming they are uninfected they continue to engage in high-risk sexual behaviour [12]. Moreover, primary HIV infection can cause mucosal ulceration and, due to the disrupted mucosal barrier, HIV transmission risk is further increased. In our case the patient had peri-anal ulceration possibly due to the primary HIV infection (genital herpes and syphilis were all excluded by negative nucleic acid amplification test results for HSV-1 and 2 and Treponema pallidum on ulcer swabs). Both ulcerative and non-ulcerative STI further increase the risk of HIV transmission two- to five-fold $[13,14]$. Our patient also had a rectal co-infection with a multi-resistant $N$. gonorrhoeae strain with reduced susceptibility to third generation parenteral cephalosporins. We recently reported on a disquieting increase of $N$. gonorrhoeae strains with reduced susceptibility to cephalosporins among MSM [15]. Parenteral cephalosporins are recommended for N. gonorrhoeae in most countries nowadays. Further susceptibility reduction towards resistance for cephalosporins would set a serious limitation in available therapy options.

Primary HIV infections are often missed or misdiagnosed as common flu, since HIV viraemia can affect all organs and cause a wide variety of non-specific symptoms like fever, fatigue, exanthema and mucosal ulceration, diarrhoea and airway infection. Hence primary care physicians should be alert for a possible primary HIV infection in patients involved in high risk behaviour, especially if they have influenza-like symptoms.
Single antibody HIV tests in particular can be false negative in primary HIV infections, as it can take on average three to four weeks before HIV antibodies become detectable (the window-phase), as shown again in this case $[16,17]$. It is therefore generally advised to repeat serologic testing for both HIV and syphilis three months after the last high risk sexual contact. For the detection of a symptomatic primary HIV infection, antibody testing alone is insufficient. Combined HIV antigen/antibody assays reduce the diagnostic window period by circa six days compared to single antibody testing alone [18]. The addition of a Nucleic Acid Amplification Test (NAAT) for HIV RNA to an HIV screening algorithm increases the identification of infected cases with 3.9 percent compared to single antibody testing using enzyme linked assays (EIA) as shown by Pilcher et al. [19]. Data on cost-effectiveness are needed before implementation of HIV RNA NAAT assays in screening algorithms is effected. Apart from increased expenses of HIV screening, NAAT testing might add to the turnaround time for test results [20]. A combined HIV antigen/antibody assay as part of a screening algorithm is a good alternative which detects approximately $90 \%$ of the acute HIV infections compared to HIV RNA detection in a screening algorithm [21]. Presently we are introducing a rapid combined HIV antigen/antibody assay for screening purposes in our clinic. In cases suspected of symptomatic primary HIV infection with a negative combined HIV antigen/antibody combined assay result, HIV-RNA testing and/or repeated combined HIV testing is advisable.

\section{References}

1. Heijman TL, Van der Bij AK, De Vries HJ, Van Leent EJM, Thiesbrummel HF, Fennema HS. Effectiveness of a risk-based visitor-prioritizing system at sexually transmitted infection outpatient clinic. Sex Transm Dis. 2007;34(7):50812.

2. Koek AG, Bruisten SM, Dierdorp M, van Dam AP, Templeton K. Specific and sensitive diagnosis of syphilis using a real-time PCR for Treponema pallidum. Clin Microbiol Infect. 2006;12(12):1233-6.

3. Bruisten SM, Cairo I, Fennema H, Pijl A, Buimer M, Peerbooms PG, et al. Diagnosing genital ulcer disease in a clinic for sexually transmitted diseases in Amsterdam, The Netherlands. J Clin Microbiol. 2001;39(2):601-5.

4. Pilcher CD, Tien HC, Eron JJ Jr, Vernazza PL, Leu SY, Stewart PW, et al. Brief but efficient: acute HIV infection and the sexual transmission of HIV. J Infect Dis. 2004;189(10):1785-92.

5. Yerly S, Vora S, Rizzardi P, Chave JP, Vernazza PL, Flepp M, et al. Acute HIV infection: impact on the spread of HIV and transmission of drug resistance. AIDS. 2001;15(17):2287-92.

6. Daar ES, Moudgil T, Meyer RD, Ho DD. Transient high levels of viremia in patients with primary human immunodeficiency virus type 1 infection. N Engl J Med. 1991;324(14):961-4.

7. Clark SJ, Saag MS, Decker WD, Campbell-Hill S, Roberson JL, Veldkamp PJ, et al. High titers of cytopathic virus in plasma of patients with symptomatic primary HIV-1 infection. N Engl J Med. 1991;324(14):954-60.

8. Pilcher CD, Shugars DC, Fiscus SA, Miller WC, Menezes P, Giner J, et al. HIV in body fluids during primary HIV infection: implications for pathogenesis, treatment and public health. AIDS. 2001;15(7):837-45.

9. Tindall B, Evans L, Cunningham P, McQueen P, Hurren L, Vasak E, et al. Identification of HIV-1 in semen following primary HIV-1 infection. AIDS. 1992;6(9):949-52.

10. Pinkerton SD. Probability of HIV transmission during acute infection in Rakai, Uganda. AIDS Behav. 2008;12(5):677-84.

11. Wawer MJ, Gray RH, Sewankambo NK, Serwadda D, Li X, Laeyendecker 0, et al. Rates of HIV-1 transmission per coital act, by stage of HIV-1 infection, in Rakai, Uganda. J Infect Dis. 2005;191(9):1403-9.

12. Bezemer D, de Wolf F, Boerlijst MC, van Sighem A, Hollingsworth TD, Prins M, et al. A resurgent HIV-1 epidemic among men who have sex with men in the era of potent antiretroviral therapy. AIDS. 2008;22(9):1071-7.

13. HIV prevention through early detection and treatment of other sexually transmitted diseases--United States. Recommendations of the Advisory Committee for HIV and STD prevention. MMWR Recomm Rep. 1998;47(RR-12):1-24. 
14. Fleming DT, Wasserheit JN. From epidemiological synergy to public health policy and practice: the contribution of other sexually transmitted diseases to sexual transmission of HIV infection. Sex Transm Infect. 1999;75(1):3-17.

15. de Vries HJ, van der Helm JJ, Schim van der Loeff MF, van Dam AP. Multidrugresistant Neisseria gonorrhoeae with reduced cefotaxime susceptibility is increasingly common in men who have sex with men, Amsterdam, the Netherlands. Euro Surveill. 2009;14(37):pij=19330. Available from: http://www. eurosurveillance.org/ViewArticle. aspx?ArticleId $=19330$

16. Kwon JA, Yoon SY, Lee CK, Lim CS, Lee KN, Sung HJ, et al. Performance evaluation of three automated human immunodeficiency virus antigen-antibody combination immunoassays. J Virol Methods. 2006;133(1):20-6.

17. Fiebig EW, Wright DJ, Rawal BD, Garrett PE, Schumacher RT, Peddada L, et al. Dynamics of HIV viremia and antibody seroconversion in plasma donors: implications for diagnosis and staging of primary HIV infection. AIDS. 2003;17(13):1871-9.

18. Sickinger E, Stieler M, Kaufman B, Kapprell HP, West D, Sandridge A, et al. Multicenter evaluation of a new, automated enzyme-linked immunoassay for detection of human immunodeficiency virus-specific antibodies and antigen. J Clin Microbiol. 2004;42(1):21-9.

19. Pilcher CD, Fiscus SA, Nguyen TQ, Foust E, Wolf L, Williams D, et al. Detection of acute infections during HIV testing in North Carolina. N Engl $\mathrm{J}$ Med. 2005;352(18):1873-83.

20. Centers for Disease Control and Prevention (CDC). Acute HIV infection - New York City, 2008. MMWR Morb Mortal Wkly Rep. 2009; 58(46):1296-1299.

21. Fiscus SA, Pilcher CD, Miller WC, Powers KA, Hoffman IF, Price M, et al. Rapid, real-time detection of acute HIV infection in patients in Africa. J Infect Dis. 2007;195(3):416-24 